\title{
Towards Quantitative Atomic-Scale Imaging of Magnetization Distributions in Materials Using Aberration-Corrected Off-Axis Electron Holography
}

\author{
Rafal E. Dunin-Borkowski ${ }^{1}$, Jan Caron ${ }^{1}$, Patrick Diehle ${ }^{1}$, Amir H. Tavabi ${ }^{1}$ and András Kovács ${ }^{1}$ \\ 1. Ernst Ruska-Centre for Microscopy and Spectroscopy with Electrons and Peter Grünberg Institute, \\ Forschungszentrum Jülich, Jülich, Germany.
}

Off-axis electron holography is a powerful technique for recording the phase shift of a high-energy electron wave that has passed through an electron-transparent specimen in the transmission electron microscope. The phase shift is, in turn, sensitive to the electrostatic potential and magnetic induction in the specimen, projected in the electron beam direction. When using the technique to record local information about magnetic fields in nanoscale materials, the desired magnetic contribution to the phase shift must usually be separated from the unwanted and often dominant electrostatic contribution, which can be associated with local changes in composition, sample thickness, dynamical diffraction and longer-range charge redistribution. In addition, as the magnetic contribution to the phase shift can be very weak, the acquisition of multiple holograms and the subsequent alignment and averaging of the resulting phase images is often necessary. Here, we describe two further improvements to the application of off-axis electron holography to the characterization magnetic materials, which we are currently pursuing: the use of a model-based algorithm to infer magnetization distributions quantitatively from recorded phase images and the prospect of using chromatic aberration correction to improve the spatial resolution of measured magnetic signals to the atomic level.

The determination of the local magnetization in a specimen from a phase image recorded using off-axis electron holography is a highly challenging ill-posed problem. We have developed a model-based iterative reconstruction technique, which can be used to retrieve the projected in-plane magnetization distribution from the magnetic contribution to a recorded phase image, or alternatively the threedimensional magnetization distribution from two, optimally orthogonal, tilt series of magnetic phase images [1]. Our approach involves the optimized implementation of a forward model, which utilizes sparse-matrix multiplications for efficient projections and subsequent fast-Fourier-transform-based convolutions with pre-calculated convolution kernels based on analytical solutions for the magnetic phase images of simple geometrical objects. The ill-posed problem is tackled by first replacing the original problem by a least squares minimization, which is augmented by regularization to find a unique solution for the reconstructed magnetization distribution. Tikhonov regularization of first order is used to apply a smoothness constraint to the magnetization, which is justified by the minimization of exchange energy. A priori information about the positions and sizes of magnetic objects within the field of view can be incorporated in the form of a three-dimensional mask, which reduces the number of unknowns to be retrieved. The approach can account for arbitrary linear phase ramps and phase offsets, as well as for untrustworthy (low confidence) regions in phase images. Diagnostic measures can be used to analyze the quality of the reconstruction results, while the chosen regularization strength can be optimized to obtain the best solution in the presence of noise. Figure 1 shows a reconstruction of the three-dimensional magnetization distribution in a disc that supports a magnetic vortex state generated from two tilt series of simulated magnetic phase images. Highly encouraging experimental results have been obtained from the reconstruction of the projected magnetization distributions of magnetic skyrmions examined in extended films and geometrically-confined structures [2], and from the reconstruction of three-dimensional magnetization distributions in sub-100-nm magnetite nanocrystals. 
In the most recent generation of transmission electron microscopes, chromatic aberration correction can be used to provide improved spatial resolution and interpretability when compared with the use of spherical aberration correction alone. The reduced dependence of image resolution on energy spread in a chromatic aberration corrected microscope offers benefits for conventional bright-field and dark-field imaging as a result of the decreased influence of inelastic scattering on spatial resolution, even when using zero-loss energy filtering. Less refocusing is necessary when moving between regions of different specimen thickness, while for energy-filtered transmission electron microscopy chromatic aberration correction allows large energy windows and large objective aperture sizes to be used without compromising the spatial resolution of energy-loss images. We are presently undertaking an experimental and theoretical assessment of the benefit of applying combined chromatic and spherical aberration correction to the Lorentz lens of the Titan PICO electron microscope in Forschungszentrum Jülich for magnetic-field-free imaging with the conventional microscope objective lens switched off. At present, we have achieved a spatial resolution of approximately $0.4 \mathrm{~nm}$ in magnetic-field-free conditions and are working to improve this value further. We are recording high-resolution phase images of magnetic oxides and hard magnetic materials in magnetic-field-free conditions. We are also carrying out computer simulations, in order to choose the optimal specimen and microscope parameters that can be used to enhance the weak magnetic contribution to an high-resolution phase image and to separate it from the unwanted, much stronger compositional and structural contribution [3].

\section{References:}

[1] J Caron, Ph.D. dissertation, RWTH Aachen University (2017).

[2] D Song et al., Phys. Rev. Lett. (2018), in press.

[3] The research leading to these results received funding from the European Research Council under the European Union's Seventh Framework Program (FP7/2007-2013)/ ERC grant number 320832. We are grateful to J Ungermann, Z-A Li, H Du, K Shibata, Y Tokura, M Riese, M Farle, N Kiselev, S Blügel, J Barthel, P Hartel, M Bischoff and A T Boothroyd for contributions to this work.
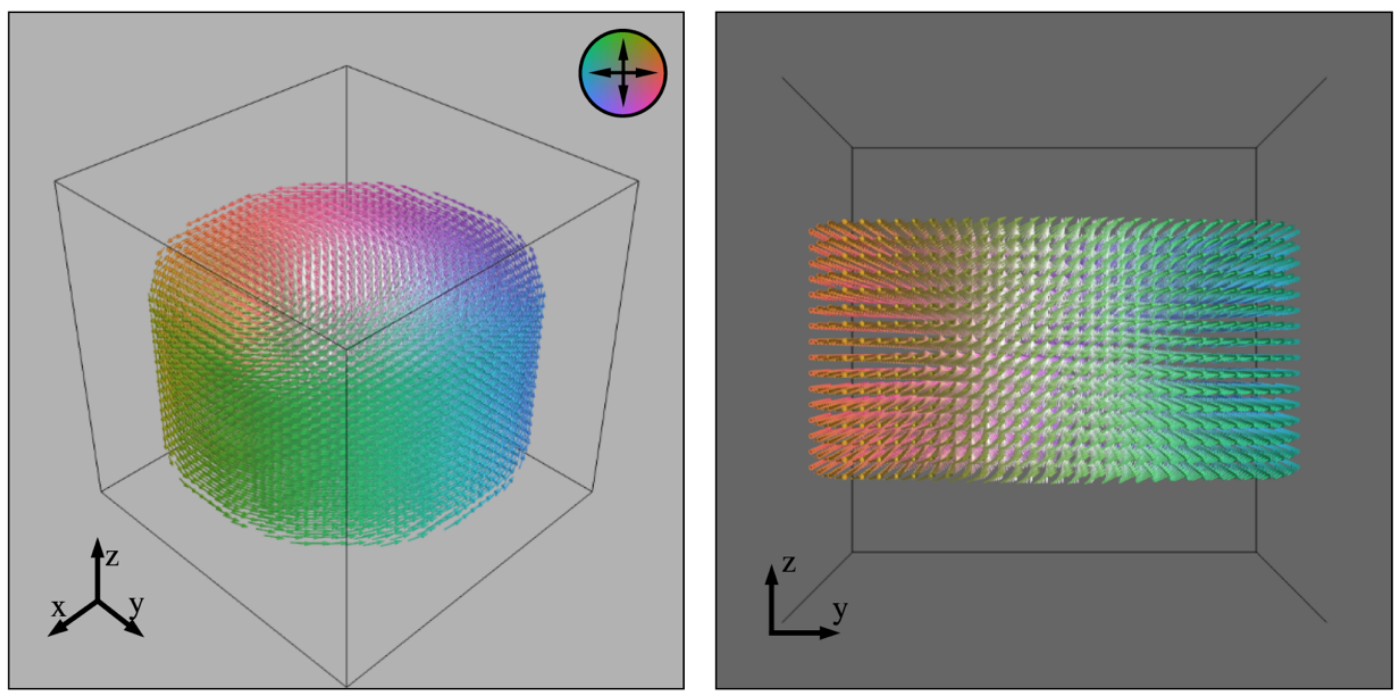

Figure 1. Reconstructed magnetization distribution of a magnetic vortex state calculated from two tilt series of simulated magnetic phase images generated for maximum tilt angles about the $\mathrm{x}$ and $\mathrm{y}$ axes of $\pm 90^{\circ}$ and an angular sampling of $5^{\circ}$. The color wheel encodes the magnetization direction in the $x y$ plane, while white arrows point in the positive $\mathrm{z}$ direction. 